\title{
Molecular material in the Bubble of the Galactic Center
}

\section{Miguel Angel Requena Torres and Alberto Noriega-Crespo}

\author{
Space Telescope Science Institute \\ San Martin Drive, Baltimore, United States of America \\ email: mrequena@stsci.edu
}

\begin{abstract}
The Galactic Center (GC) is one of the more extreme environments in the Galaxy. The so call Bubble of the GC presents structures that do not match with the general behavior of the inner Central Molecular Zone (CMZ). In this work we study the molecular emission related to the observable ionized gas emitted by this Bubble. We find dense pockets of gas and bubble like structures in the velocity domain.
\end{abstract}

Keywords. Galaxy: center, molecular data, ISM: bubbles

\section{Introduction}

The Galactic Center (GC) Central Molecular Zone is a heavily observed concentration of molecular gas, dust and ionized material. Recent studies show that most of the material is distributed in some orbits or streamers (Molinari et al. 2004, Henshaw et al. 2016, kruijssen et al. 2015). Out of this general trend, the material of the so call Bubble of the GC (Sofue 2003), that includes The Sickle, and the Thermal Arched Filaments (TAF) seems to have been driven by an energetic phenomenon in the past. Large velocities and bubble like structures can be seen in the continuum emission. Recently, The HiGAL team, interpreted some of the peaks of emission in the multi-wavelength continuum in the region as very embedded Young Stellar Objects (YSO).

\section{Overview}

In this on-going work, we study the molecular emission related to the velocity structures from the bubble regions. Disentangling the origin of molecular emission is quite complicated, but we know the velocity structure of the region due to the large number of previous observations in the area. The velocities related to the ionized gas seen in the continuum images, can be reconstructed using ionized lines from Herschel (HEXGAL key project with Herschel), or recombination lines observed previously (Simpson et al. 2004).

First, we compare the emission from warm dust and ionized lines with those of molecular material (see central panel in Fig. 1). These, do not show a very good correspondence, except in the region between the Sickle and the Arches Cluster where there is a overabundance of emission from both lines.

Using the CO molecule emission collected by IRAM and APEX telescopes, we compare the observed emission with a set of models derived using the LVG code RADEX. In the lower panel from Fig. 1, we show the complicated spectra from the main regions in The Sickle and the TAF. From the 45 sources selected by the continuum data, 11 of them show densities of the order of $10^{5} \mathrm{~cm}^{-3}$. One of the interesting things of the molecular data is that we can study the velocity information from the different components along the line of sight to try to organize the material that is emitting it. In Fig. 1, we can 


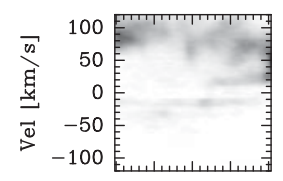

[1]

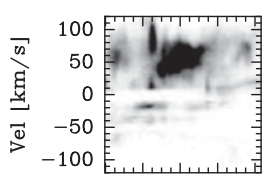

[2]

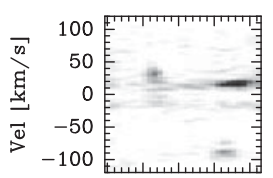

[3]

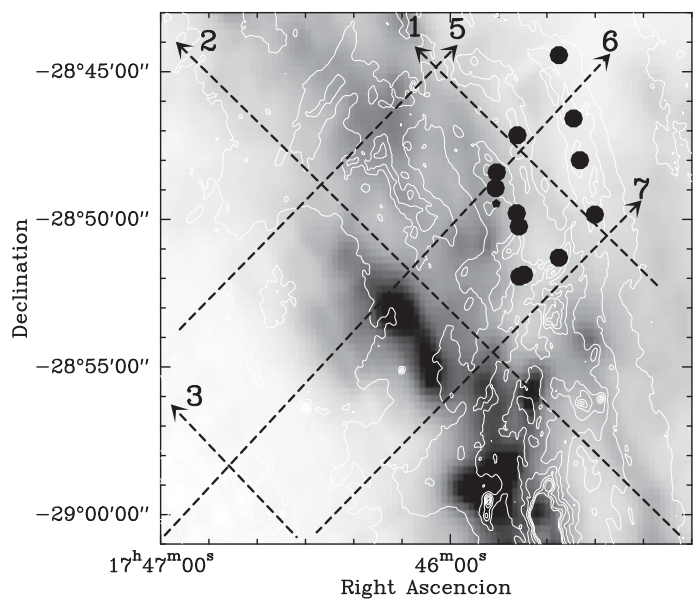

Sickle

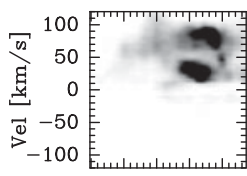

[4]

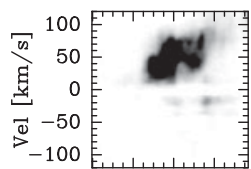

[5]

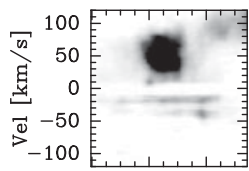

[6]

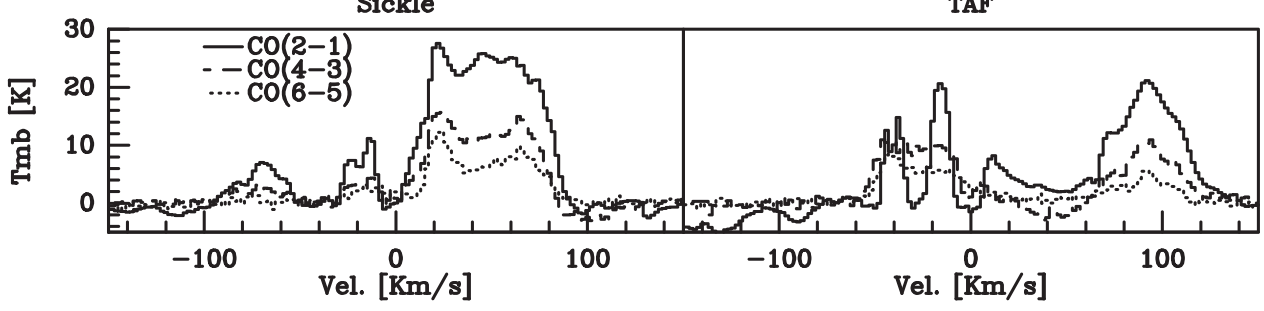

Figure 1. Central panel: Emission of the HCN molecular tracer on the Bubble region. The PACS $70 \mu$ memission is over plotted. With red points, we mark the densest cores derived with the Radex analysis from the Molinari et al. sample of possible YSOs. The dashed lines highlight the areas selected to show the velocity cuts shown in the side panels, the cuts are labeled with numbers to localize the PV diagrams. In the panels at right and left, we show the cuts in velocity of the regions in the central panel. Bottom panel:Spectral profile of two positions in the Sickle (left) and the TAF (right). The plots show the emission from some of the CO transitions used in this study to extract the physical conditions of the possible YSO observed in the continuum.

see several cuts in velocity in the area, and in the position-velocity plots we can see the different parts of the so-called bubble.

\section{Implications}

We have identified at least 11 compact structures, based on the above data, with densities high enough as to harbor in their interior very dense ("protostar-like") objects. On the large scale, some of the molecular material does show bubble-like structures in velocity space, and we intend to characterize their properties with the help of the SCOUSE code (Henshaw et al. 2016). The code's algorithm allows to better identify streams of material based on the available velocity information.

\section{References}

Molinari, S., Bally, J., Noriega-Crespo, A., et al. 2011, ApJ, 735, 33

Henshaw, J. D., Longmore, S. N., Kruijssen, J. M. D., et al. 2016, MNRAS, 457, 2675

Kruijssen, Dale, \& Longmore 2015, MNRAS, 447, 1059

Simpson, J. P., Rubin, R. H., Colgan, S. W. J., et al. 2004, ApJ, 611, 338

Sofue, Y. 2003, PASJ, 55, 445 